\title{
CORRELATIVE ANALYSIS OF VITAMIN D STATUS IN PEOPLE SUFFERING FROM TYPE-2 DIABETES MELLITUS IN EASTERN REGION OF NEPAL
}

\author{
Jha $R^{1^{*}}$, Mishra $M K^{2}$, Mishra $A^{3}$, Chaudhary $R K^{4}$
}

\author{
Affiliation \\ 1. Department of Internal Medicine, Birat Medical Collegeand \\ Teaching Hospital (BMCTH), Biratnagar \\ 2 Department of Prosthodontics, Chitwan Medical College, \\ Bharatpur \\ 3 Emergency Department, Government District Hospital, Udaypur \\ 4 Department of Human Anatomy, BMCTH, Biratnagar
}

\section{ARTICLE INFO \\ Article History \\ Received : 28 October, 2018 \\ Accepted : 1 December, 2018 \\ Published : 31 December, 2018}

(C) Authors retain copyright and grant the journal right of first publication with the work simultaneously licensed under Creative Commons Attribution License CC - BY 4.0 that allows others to share the work with an acknowledgment of the work's authorship and initial publication in this journal.

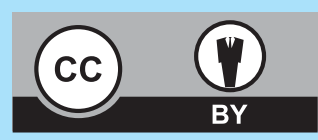

ORA 91

DOI: $10.3126 /$ bjhs.v3i3.22191

\section{* Corresponding Author}

Dr. Rajneesh Jha

Department of Internal Medicine

Birat Medical College and Teaching Hospital, Biratnagar Email:jha_rajneesh@yahoo.com

ORCID: https://orcid.org/0000-0003-4921-7801

\section{Citation}

Jha R, Mishra MK, Mishra A, Chaudhary RK. Correlative Analysis of Vitamin D Status in People Suffering from Type-2 Diabetes Mellitus In Eastern Region of Nepal. BJHS 2018;3(3)7: 565-569.

\section{ABSTRACT}

\section{Introduction}

Vitamin D deficiency exists as a common problem among population worldwide. The deficient vitamin D level leads to direct impact on various normal functioning of human body systems. It has been observed in few studies that decreased circulating concentration of 25-hydroxyvitamin $D$ is associated with type-2 diatetes mellitus. The possible reason behind such occurrence is due to the direct effect of vitamin $D$ on glucose metabolism. Vitamin D exerts influences on activity of pancreatic $\beta$-cell function from where insulin is secreted. In addition, the patients suffering from low vitamin $D$ status show insulin resistance. These affected individuals with low vitamin D status exhibits impaired markers of glucose metabolism such as glycosylated hemoglobin. So, researchers have found vitamin D deficiency to correlate with type-2 diabetes and $80 \%$ of obese adults suffering from vitamin D insufficiency state. In spite of such crucial significance, the correlative studies related to vitamin D status and type-2 diabetes still remains obscure in eastern region of Nepal.

\section{Objective}

We aimed to investigate status of vitamin D among type-2 diabetics and analyze its possible correlation

\section{Methodology}

In the present study which was hospital-based and crosssectional carried out in the Department of internal medicine of Birat Medical College Teaching Hospital from Junuary 2018 to June 2018, antecubital venous blood samples were collected from patients $(n=100)$ in plain vials with informed written consent. Blood samples were allowed to clot and centrifuged for separation of serum. The separated sera were further processed for determination of glucose (fasting as well as post-prandial) by spectrophotometry and estimation of vitamin D with use of microwell Enzyme-linked immunosorbant assay (ELISA) technique. The data was interpreted by using SPSS software version 16 .

\section{Results}

We found that $75 \%$ type-2 diabetics had suffered from "state of vitamin D deficiency". Among them, females, in contrast, were predominating which was interesting in an analytical point of view.

\section{Conclusion}

Our study reviews evidence on disorders related to type-2 diabetes mellitus and vitamin D status, especially in older people. The biological processes that lead to synthesis of vitamin $D$ in human body tissues become less efficient when an individual's age advances with in additional occurrences of type- 2 diabetes mellitus. We should therefore maintain the repletion of vitamin $D$ in healthy older people via supplementary intakes and sensible sun-exposure.

\section{KEY WORDS}

Diabetes, Deficiency, Vitamin D 


\section{INTRODUCTION}

The vitamin $D$ is accessible in natural foods meaning that humans should consume them on regular basis. In addition to their availability in natural foods, human body tissues produce vitamin $\mathrm{D}$ in response to exposure of sunlight. ${ }^{2}$ Vitamin D deficiency, however, exist as common problem in population. ${ }^{3}$. The deficient level of vitamin $D$ exhibits direct impact on normal functioning of human body systems. Bones and tendons require sufficient amount of vitamin $D$ as normal growth requirement. ${ }^{4}$ The reduced level of 25 hydroxyvitamin $\mathrm{D}$ is associated with type-2 diatetes mellitus. The already known fact behind this occurrence is due to the direct effect of vitamin $D$ on glucose metabolism. Vitamin D exerts influences on activity of pancreatic $\beta$-cell function from where insulin is secreted. These affected individuals with low vitamin $D$ status exhibits impaired $\mathrm{HbA}_{1} \mathrm{C}$. The inadequacy of vitamin $\mathrm{D}$, further, retards physical growth with a risk in fracture of hip bones among elderly people. ${ }^{5}$ Maintenance of normal levels of vitamin D, therefore, is essential for appropriate regulation of systemic processes. Thus, vitamin D deficiency is emerging as a serious health problem. Hence, estimation of its plasma levels is essential for determining the grade of an individual who may stand either under-deficiency or over-nutrition state. As it transforms metabolic processes of glucose and deficiency is worldwide being prevalent in stages that range from degree of newborn to infancy and adulthood, determination of vitamin $D$ is crucial. ${ }^{6}$

Moreover, numerous studies have reported for existence of higher incidences of vitamin $D$ deficiency among type-2 diabetics.' So, continuation of vitamin $D$ deficiency may affect type-2 diabetes control. Vitamin D deficiency typically associates with worsening glycemic index and increased insulin requirements. ${ }^{8}$ As per the data put forwarded by World Health Organization, a pandemic disease called diabetes mellitus affects 170 million patients with having an effect on more than double to 370 million patients by year 2030. Researchers, in contrast, have found vitamin $D$ deficiency to correlate with type- 2 diabetes and $80 \%$ of obese adults suffering from vitamin D insufficiency state. ${ }^{9}$ Scientists have shown low vitamin D status to associate with development of type-2 diabetes as well as metabolic syndrome so that these consequences reflect as incidences of vital clinical importance. ${ }^{10}$ In spite of suchcrucial significance, the correlative studies related to vitamin $D$ status and type-2 diabetes still remains obscure among type-2 diabetics s in eastern Nepal.

\section{METHODOLOGY}

\section{Study design and enrolment criteria:}

This was a hospital-based, cross-sectional correlative study carried out in the Department of Internal Medicine with Diagnostic Laboratory section at Birat Medical College and Teaching Hospital (BMCTH), Tankisinuwari, Biratnagar, Morang, Nepal from January 2018 to June 2018. A total of 100 type-2 diabetic patients with age group ranging from 20 and 60 years-old were registered to meet the target population. Exclusion criteria were: patients using medications such as anticonvulsions, antitubercular drugs, calcitonin, gabapentin, corticosteroids and patients diagnosed with diseases like osteoporosis, osteomalacia, rheumatoid arthritis. In addition to estimation of their glucose levels, these patients were investigated for vitamin D status in collected blood samples. The informed written consent was taken from all patients and study was approved by Institutional Ethical Committee. All the participants had undergone detailed history, clinical examination and laboratory investigations using Performa designed for this study.

All patients were subjected to following investigations: determination of fasting blood sugar (FBS) and post-prandial blood sugar (PPBS) as well as estimation of vitamin D.

\section{Sample collection and serum preparation:}

Venipuncture was performed to collect blood samples under universal attentiveness as described in manufacturer's protocol. ${ }^{11}$ Antecubital venous blood was collected from patients in plain vials with informed written consent, strictly as per the norms recommended by Institutional Ethical Committee. Blood samples were allowed to clot for five min and centrifuged at $3000 \mathrm{rpm}$ for $10 \mathrm{~min}$ in order to separation of serum. Sera were stored at- $20^{\circ} \mathrm{C}$ until assessment. All steps were carried out under sterile conditions and precautions were taken to prevent blood samples from hemolysis, as described earlier. ${ }^{12-13}$

\section{Determination of glucose in serum:}

The determination of glucose levels in serum was done using spectrophotometer and glucose oxidase-peroxidase assay kit by Tinder's method. ${ }^{14}$ The unhemolysed serum samples and normal as well as abnormal controls were used while estimation, according to the instructions provided by manufacturers.

\section{Estimation of vitamin D:}

Competitive immunoassay was performed for estimation of serum levels of vitamin $D$ using direct chemiluminescent technology with ADVIA Centaur vitamin D assay kit. The ADVIA Centaur XP system was utilized while performing assay. The system was prepared after loading reagent packs containing anti-vitamin D-antibody. Instrument automatically performed certain actions that include: dispensing $100 \mu \mathrm{l}$ serum into cuvette, adding $115 \mu \mathrm{l}$ dithiothreitol, $200 \mu \mathrm{l}$ solid-phase reagent followed by incubation at $37{ }^{\circ} \mathrm{C}$ for 5 min and administering $200 \mu \mathrm{l}$ lite reagent with incubation at $37^{\circ} \mathrm{C}$ for $2.5 \mathrm{~min}$. Then machine had separated, aspirated and washed the cuvettes with reagent water. Afterwards instrument had dispensed $300 \mu$ leach of acid and base reagents to initiate chemiluminescent reaction. The patients were declared deficient if they demonstrated serum levels of vitamin $D<10$, insufficient when concentrations resided between 10-30, sufficient after values $>30$ and toxicity if $>100 \mathrm{ng} / \mathrm{ml}$. Results were obtained according to selection option in system. Based on setting up of the assay, system had reported serum levels of vitamin D in terms of $\mathrm{ng} / \mathrm{ml}$.

\section{Data interpretation:}

The validity and reliability of test results were determined using control sera (from BIO-RAD). Data were analyzed 
under Software Package for Social Science version 16 (SPSS 16). RESULTS

\section{Strategy to investigation of vitamin D status among type-2} diabetic patients: Strictly as per the rules prescribed by American Diabetic Association and guidelines provided by World Health Organization, all participants had undergone through detailed history and clinical examination. ${ }^{15} \mathrm{~A}$ total of 100 patients with age-groups ranging from 20- to 60years-old were enrolled for investigation. All the patients were subjected to investigation for FBS and PPBS. These individuals, if having FBS $\geq 7.1 \mathrm{mmol} / \mathrm{L}$ (or $126 \mathrm{mg} / \mathrm{dl}$ ) and PPBS $\geq 15.2 \mathrm{mmol} / \mathrm{L}$ (or $200 \mathrm{mg} / \mathrm{dl}$ ), were registered as type-2 diabetics ( $1^{\text {st }} \& 2^{\text {nd }}$ bars, respectively, in Figures $1 \& 2$ ) in present study. All of them were then subjected to further investigation for vitamin D status.

Diabetics had wide range of circulating vitamin D levels, but no toxicity: In a total 100 type- 2 diabetics, we found values of FBS and PPBS ranging as 7.2-8.3 and 15.2-18.6 $\mathrm{mmol} / \mathrm{L}\left(1^{\text {st }} \& 2^{\text {nd }}\right.$ bars in Figures $\left.1 \& 2\right)$, respectively, and so these had been the target population. Further, our data demonstrated that they had their circulating levels of vitamin D ranging from 8 and $59 \mathrm{ng} / \mathrm{ml}$ ( $3^{\text {rd }}$ bar; Figure-1); suggestive of a fact that such patients had wide variation in circulating levels vitamin D. In contrast, none of them had relatively shown the values $>100 \mathrm{ng} / \mathrm{ml}$ (data not shown); indicative to absence of vitamin D toxicity among these type2 diabetic individuals.

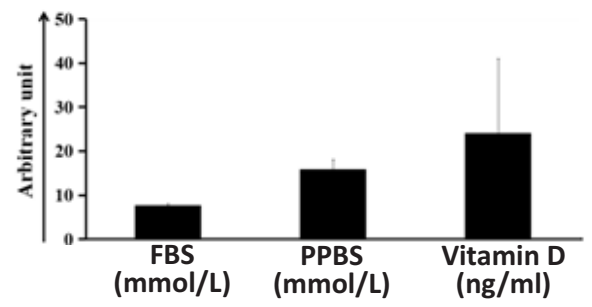

Figure 1: Bar diagram demonstrating serum levels of FBS, PPBS \& vitamin D in diabetic individuals $(n=100)$

Majority of type-2 diabetics suffered from "the state of vitamin D deficiency":

With respect to above finding on demonstration of a wide variation in vitamin D status in type-2 diabetics, population was then grouped based on their vitamin $D$ levels. In contrast as serum levels of vitamin $D<30 \mathrm{ng} / \mathrm{ml}$ require corresponding supplementation under therapeutic measure, we reasoned to consider both vitamin D-deficient and -insufficient groups (with $\mathrm{D}<10$ and between 10-30 $\mathrm{ng} / \mathrm{ml}$, respectively) into a single set as under "the state of vitamin D deficiency" and those having > $30 \mathrm{ng} / \mathrm{ml}$ to subsist in normal regime.

With above postulation and among 100 number of total type-2 diabetics, we found that 25 people had vitamin $D$ levels in normal limit i.e., $>30 \mathrm{ng} / \mathrm{ml}$ ( $3^{\text {rd }}$ bar; Figure-2). Remarkably the remaining large group $(n=75)$ had vitamin $D<30$ ( $4^{\text {th }}$ bar; Figure-2); suggesting that majority (means $75 \%$ ) of type-2 diabetics in locality suffered from "the state of vitamin D deficiency".

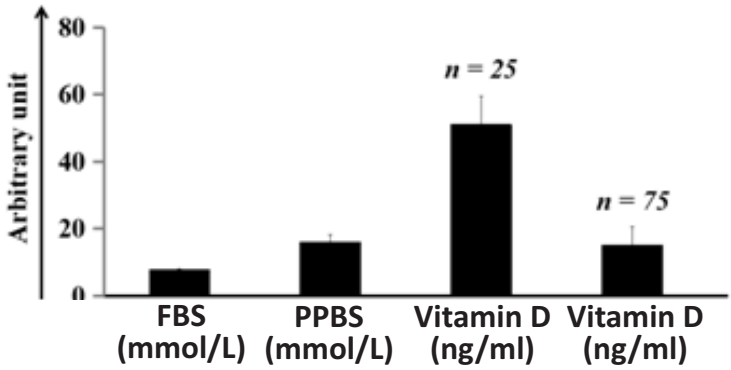

Figure 2: Bar diagram showing differential status of vitamin $D$ in type-Il diabetics ( $n=100) ; 25$ persons showed normal vitamin D levels as $51.04 \pm 8.48$ (i.e., $>30 \mathrm{ng} / \mathrm{ml}$ ) and residual 75 remained in a deficiency regime with $15.12 \pm 5.46$ (i.e., $<30 \mathrm{ng} / \mathrm{ml}$ )

Correlation of vitamin D status among type-2 diabetics with progression of age: we divided $75 \%$ of type- 2 diabetics (who suffered from "the state of vitamin D deficiency") into four sets like 'A', 'B', 'C' \& 'D' consisting of individuals as 2130, 31-40, 41-50 \& 51-60 years-old, respectively.

Out of total type-2 diabetics who suffered from state of vitamin D deficiency $(n=75)$, eight individuals stood in agegroup of 21-30 years-old ( $1^{\text {st }}$ bar; Figure-3). Prevalence, therefore, remained approximately $10 \%$ type- 2 diabetics in this ' $A$ ' set; indicative to lesser but significant existence of vitamin $D$ deficiency in these victims. Further, 11 of 31-40 years-old type- 2 diabetics ensured vitamin D deficiency $\left(2^{\text {nd }}\right.$ bar; Figure-3). Further vitamin D deficiency appeared in 19 diabetics with age-group of $41-50$ years-old ( $3^{\text {rd }}$ bar; Figure-3), suggesting that generality was $25 \%$. Interestingly when we had look upon a residual group ' $D$ ' holding the type-2 diabetics with 51-60 years-old, immensely around $50 \%$ ( $n=37$ ) were patients ( $4^{\text {th }}$ bar; Figure-3), suggestive of predominant existence of vitamin $D$ deficiency.

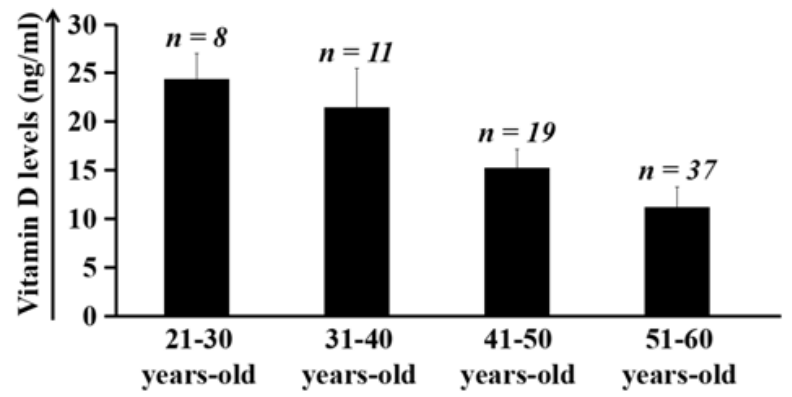

Figure 3: Bar diagram showing variation of vitamin D levels in diabetics who were in deficiency state $(n=75)$ as age-wise; ' $n$ ' indicates the number of diabetics suffered from vitamin $D$ deficiency.

Vitamin D deficiency was comparatively $60 \%$ prevalent in old-aged-women: In the present survey carried out by us in eastern Nepal , our data showed 50 \% prevalence in 51-60 years-old type-2 diabetics towards vitamin D deficiency. We, therefore, investigated for probability of significant changes in distribution between males and females. Here when we separated the subjects $(n=37)$ depending on sex and who were $>50$-years-old, around $40 \%(n=15)$ persons were male and female counterpart was dominating with $60 \%(n=22)$ prevalence (Figure-4 and pie-chart in Figure-4). 


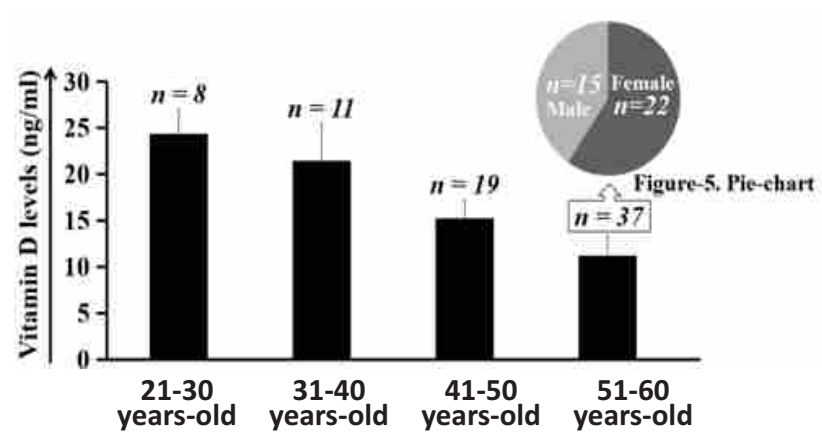

Figure 4: Bar diagram showing variance of vitamin D level and Pie chart to demonstrate gender-based distribution of 51-60 years old local diabetics.

\section{DISCUSSION}

In present study, we observed that majority ( $75 \%$ ) of type2diabetics who attended the hospital in eastern part of Nepal suffered from "state of vitamin D deficiency" (Figure 2). Based on few studies carried out previously in relative perspective, vitamin $D$ deficiency was shown to be more common among type-2 diabetics. ${ }^{16-18}$ Our observation stands in accordance with these reports. By contrast, in eastern part of Nepal, frequency of vitamin D status remained unknown. Thus we screened type- 2 diabetic individuals to investigate existence of associated status of vitamin $D$ and observed preponderant significance of vitamin D deficiency in this area (Figure 2).

Vitamin $\mathrm{D}$ deficiency exists as a common clinical problem these days. According to the survey carried out by Martina R., more than half of the adult people in Germany had vitamin D levels below common threshold. ${ }^{19}$ Since then there had been a lot of attention for vitamin $D$ in scientific community. Other study carried out by Daga et al in North India, $91.1 \%$ of type-2 diabetic patients had vitamin D deficiency. In this study, vitamin $D$ concentrations were $7.88 \pm 1.2$ and $16.64 \pm 13.6 \mathrm{ng} / \mathrm{ml}$ in diabetic and nondiabetic patients, respectively. ${ }^{20}$ In addition, Yousef A.S. had reported about prevalence of vitamin $D$ deficiency in the Kingdom of Saudi Arabia in every age group. ${ }^{21}$ According to Colorado prevalence study, Alejandro S. had shown commonness of vitamin D inadequacy in Portuguese older adults. In such study, nationwide cluster sample of 1500 Portuguese subjects $>65$ years of age suffered from vitamin D deficiency. ${ }^{22}$ In the present study, we observed that $75 \%$ people who had suffered from type-2 diabetes mellitus stood in the state of vitamin $D$ deficiency. When we divided such $75 \%$ type-2 diabetics into four sets designated as ' $A$ ', ' $B$ ', ' $C$ ' and 'D' consisting of individuals as described above, our data laid us to note that $50 \%(n=37)$ were patients s of vitamin D deficiency ( $4^{\text {th }}$ bar, Figure-3). Our findings were therefore suggestive of predominant existence of vitamin $D$ deficiency. According to study previously carried out by Barbara J.B., hypovitaminosis D exist as common problem worldwide, but it is more common and severe in older people as we observed in our study. ${ }^{10}$
In age group 51-60years females had higher prevalence of Vitamin D deficiency as compared to male. This has been the striking prevalence among old-aged type-2 diabetics. In this analytical point of view to discuss, Hanan A.H. had also obtained the similar data and so had reported the fact that vitamin D levels were significantly higher than females. ${ }^{23}$ These findings, in the study carried out by Hanan A.H., had been suggestive to the fact that vitamin $D$ levels were affected by age, nationality, gender and health statues. In beneficial point of view to the patients having vitamin $D$ deficiency, sensible sun exposure cause health benefits and maintains the strong bones. ${ }^{24}$

\section{CONCLUSION}

Our study reviews evidence on disorders related to type-2 diabetes mellitus and vitamin D status, especially in older people. Vitamin $D$ is as essential for bone health in adults and in children to prevent osteomalacia and muscle weakness. Skin provided vitamin D synthesis followed by UVB irradiation from summer sunshine and to lesser extent via absorption from food. However, these processes become less efficient when an individual's age advances with in additional occurrences of type-2 diabetes mellitus. We should therefore maintain the repletion of vitamin $D$ in healthy older people via supplementary intakes and sensible sun exposure.

\section{RECOMMENDATIONS}

Based on our observation in the present study, we strongly recommend to supplement vitamin $D$ in both diabetic and non-diabetic older people.

\section{LIMITATION OF THE STUDY}

As the present study has been carried out in an urban city Biratnagar, it would be more applicable if the rural areas would have been included.

\section{ACKNOWLEDGEMENTS}

The authors are thankful to patients for their helpfulness without which the study would be difficult to carry out. The authors, in addition, sincerely acknowledge the Department of Diagnostic Laboratory and Institutional Board of BMCTH for needful co-operation.

\section{CONFLICT OF INTEREST}

Authors declare no conflict of interest.

\section{FINANCIAL DISCLOSURE}

None 


\section{REFERENCES}

1. Zang R, Naughton DP. Vitamin D in health and disease; current perspectives. Nutrition J. 2010; 9: 65. doi.org/10.1186/1475-28919-65

2. Weedad ZM, RA Hegazy. Vitamin D and the Skin: Focus on a complex relationship: A review. J Adv. Res. 2015; 6 (6): 793-804. doi.org/ 10.1016/j.jare.2014.01.011

3. Palacios C, Lilliana $\mathrm{G}$. Is vitamin D deficiency a major global public health problem? J. Steroid BiochemMol Biol. 2014; 144: 138-145. doi.org/10.1016/j.jsbmb.2013.11.003

4. Raewyn P, Allahdad Z, Afsie S, Philippa H. Tendon, a vitamin D-responsive tissue: Why the British weather may not just be bad for your bones. Intl J ExpPathol. 2013; 95: 20. doi: 10.1111/iep.12034

5. Lips P, Netelenbos JC. TijdschrGerontolGeriatr. 1985; 16 (6): 239-245. doi.org/10.1016/j.bone.2007.10.026

6. Daniel DB. Vitamin D Metabolism, Mechanism of Action, and Clinical Applications. Chem Biol. 2014; 21 (3): 319-329. doi.org/10.1016/ j.chembiol.2013.12.016

7. Berridge MJ. Vitamin D deficiency and diabetes. Biochem J. 2017; 474 (8): 1321-1332. DOI: 10.1042/BCJ20170042

8. Khalid SA, Somoa AB, Murtadha JK. Glycemic changes after vitamin D supplementation in patients with typ-1 diabetes mellitus and vitamin D deficiency. Ann Saud Med. 2010; 30 (6): 454-458. PMID: 21060157

9. Mohammed A, Adnan AGHA, Mohammed D. Vitamin D Deficiency in Patients with Type-2 Diabetes Mellitus in Southern Region of Saudi Arabia. 2013; 8 (3): 231-236. PMID: 24371490

10. Barbara JB. Is vitamin D status relevant to metabolic syndrome? Dermatoendocrinol J. 2012; 4 (2): 212-224. doi.org/10.4161/ derm. 20012

11. Khatib OMN. Guidelines for the prevention, management and care of diabetes mellitus. EMRO Technical Publications Series 32 . www.emro.who.int/dsaf/dsa664.pdf.

12. Mallick RL, Walavalkar SJ, Pokhrel S, Singh RK. Old-aged, adolescent and pregnant women in eastern Nepal are more prone to sub-clinical hypothyroidism: a hospital-based retrospective study. Intl J Pharm and Biol Arch. 2016; 19 (1): 1-7.

13. Walavalkar SJ, Kumar P, Das G, Mallick RL. Vitamin $B_{12}$ deficiency is more common in young women at Eastern Nepal: A hospital-based collective study. Adv Res J Med and Clin Sci. 2016; 2 (5): 1-5.
14. Thomas L. Clinical Laboratory Diagnostics, $1^{\text {st }}$ edition. Frankfurt: THBooks-15. Verlagsgesellchaft; 1998: 131-137.

15. Standards of Medical Care in Diabetes. 2011. America Diabetes Association. medcraveonline.com/JDMDC/-04-00106.pdf

16. Mohammad AB, Rogheyeh A, Bahar B, Fayyaz S. Status of vitamin D in diabetic patients. 2014. Caspian J Intl Med. 2014; 5 (1): 40-42. PMID: 24490013

17. Teresa M, Campbell RK. Vitamin D and Diabetes. Diabetes Spectrum. 2011; 24 (2): 113-118. doi.org/10.2337/diaspect.24.2.113

18. Sue KP, Cedric FG, Edward DG, Luke BD, Elizabeth BC. Plasma 25dihydroxyvitamin $\mathrm{D}$ concentration and risk of type-2 diabetes and pre-diabetes: 12-years cohort study. Plos One. 2018; 13 (4): e0193070: 10.137/journal.pone.0193070

19. Martina R, Christa SN, Markus AB, Nina R, Birte H, Gert BMM. Vitamin $D$ status among adults in Germany-results from the German Health Interview and Examination Survey for adults (DEGS1). BMC Public Health. 2015; 15: 641. doi.org/10.1186/s12889-015-2016-7

20. Daga RA, Laway BA, Shah ZA, Mir SA, Kotwal SK, Zargar AH. High prevalence of vitamin $D$ deficiency among newly diagnosed youthonset diabetes mellitus in north India. Arg Bras Endocrinol Metabol. 2012; 56 (7); 423-428. PMID: 23108746.

21. Yousef AS, Nasser MAD, Nassiruddin K, Hanan A, Abdulaziz MAO, Majed SA, George PC. Vitamin D status in Saudi school children based on knowledge. BMC Pediatr. 2015; 15: 53. doi.org/10.1186/s12887015-0369-9

22. Alejandro S, Teresa FA, Rita SG, Ana SS, Luisa A, Pedro M, Patricia P, Claudia A, Nuno B. Vitamin D status and associated factors among Portuguese older adults: results from Nutrition UP 65 cross-sectional study. Nutrition and Metabolism. 2017; 7: e016123. doi: 10.1136/ bmjopen-2017-016123.

23. Hanan A, Hani T, Shmeylan A, Saleh A, Waleed T. Vitamin D status among patients visiting a tertiary care center in Riyadh, Saudi Arabia: a retrospective review of 3475 cases. 2014; 14: 159. doi.org/ 10.1186/1471-2458-14-159

24. Kellie LSB, John B, Elizabeth LS, Colin RD, Stuart IH, Peter LT. Burning daylight: Balancing vitamin $D$ requirements with sensible sun exposure. Med J Aust. 2011; 194 (7); 345-348. doi: 10.5694/ mja2.12037 